\title{
ON THE PRESENCE OF VARIABLE STARS IN OPEN CLUSTERS
}

\author{
M. POPOVA
}

Dept. of Astronomy, Bulgarian Academy of Sciences, Sofia

\begin{abstract}
The significance of variable stars as members of open clusters is outlined. The results of a search for such stars are briefly mentioned.
\end{abstract}

The search for variable stars in open clusters is important in establishing their place in stellar evolution. Moreover, it enables the determination of their absolute magnitudes and intrinsic colours.

An extensive list of variables within 2 cluster radii was published by Kholopov (1956). In recent years such a large number of variable stars have been discovered that we decided to compile a new list of variables within 5 cluster radii. This list contains 2253 variables in 362 clusters. It will be published in the Supplement of Variable Stars. For each variable its distance from the cluster centre is given. This quantity has to be accepted as a first criterion for cluster membership, other criteria not being available.

A preliminary statistical investigation shows a significant concentration of variables towards the cluster centre. The relative density of variables with the distance from the centre in units of cluster radii is given in Table $\mathrm{I}$.

TABLE I

\begin{tabular}{llllll} 
Zone & $0<r<1$ & $1<r<2$ & $2<r<3$ & $3<r<4$ & $4<r<5$ \\
\hline $\begin{array}{c}\text { Relative } \\
\text { density }\end{array}$ & 1 & 0.47 & 0.39 & 0.32 & 0.29
\end{tabular}

Analysis of the distribution of different types of variables confirms the presence of Cepheid, irregular, eclipsing, magnetic and Mira variables in open clusters. An observing programme of individual variables being possible members of open clusters is in preparation.

\section{Reference}

Kholopov, P. N.: 1956, Variable Stars, 11, 325. 